\title{
Mechanical characterization of metal-ceramic composites
}

\author{
G. Bolzon, M.Bocciarelli, E.J. Chiarullo \\ Politecnico di Milano, Dipartimento di Ingegneria Strutturale, \\ bolzon@stru.polimi.it
}

RIASSUNTO. I compositi metallo-ceramici rappresentano una classe di materiali per uso strutturale che richiede una adeguata caratterizzazione meccanica. Le difficoltà ed i costi associati con la produzione e la lavorazione di questi compositi scoraggiano l'esecuzione di prove meccaniche tradizionali, che richiedono l'impiego di un numero statisticamente significativo di provini di dimensioni e geometria proibitivi in questo contesto. Risulta invece indicata la prova di indentazione strumentata, rapida ed economica, abbinata a tecniche di analisi inversa che combinano l'informazione sperimentale con la simulazione del comportamento del materiale durante la prova, come si mostra in questo contributo basato sull'esperienza acquisita nell'ambito del Network di Eccellenza su 'Knowledge-based Multi-component Materials for durable and safe performance' (KMM-NoE).

ABSTRACT. Metal-ceramic composites represent a class of quasi-brittle materials for advanced structural applications that require adequate mechanical characterization. Difficulties and costs associated with material production and specimen extraction prevent the execution of a statistically meaningful number of standard laboratory tests. Parameter calibration methodologies based on instrumented indentation and inverse analysis represent fast and reliable identification procedures in the present context, as shown by the present contribution, based on some experience achieved in the framework of the European Network of Excellence on 'Knowledge-based Multi-component Materials for durable and safe performance' (KMM-NoE).

KEYWORDS. Metal-ceramic composites; constitutive models; quasi-brittle fracture; mechanical characterization; instrumented indentation; parametric identification; inverse analysis.

\section{INTRODUCTION}

$\mathrm{M}$ etal-ceramic composites represent a class of advanced materials of growing interest for their application in several technological fields, ranging from energy production and biomechanics as witnessed, e.g., by the website www.kmm-noe.org. Significant dimensional stability, reduced thermal expansion, increased wear and damaging resistance at high temperature, good mechanical strength (mainly in compression) make these composites interesting replacement of metals as structural components and protective coatings in high demanding applications. Main limitations concerning their usage consist of: difficult processing; high production costs; strong influence on the overall material properties of micro-structural details; brittle behaviour, though mitigated by the metal phase when compared with pure ceramics. The composition and the production process of these composites are usually designed in order to optimize their effective thermo-mechanical response, which require to carefully control the possible presence and the evolution of local damages and defects.

The estimation of macroscopic properties is often based on homogenization techniques, which often neglect microstructural details playing a significant role for brittle and quasi-brittle materials [1-8]. A proper experimental validation of the mechanical properties is therefore mandatory. On the other hand, difficulties and costs associated with specimen production prevent the execution of a statistically meaningful number of standard laboratory investigations.

A practical methodology for material characterization is based on instrumented indentation, which represents a fast and economical test, which can be performed at different scales on small material portions, even directly on the structural 
components. The combination of experimental information with the test simulation and inverse analysis [9] represents the envisaged approach to material characterization as the properties to be determined becomes numerous and less amenable to direct separate measurement, as in the present context. This approach can return the bulk and fracture properties of metal-ceramic composites in a robust and reliable manner, by exploiting experimental data relevant to both the traditional indentation curves and the mapping of the residual displacement field, as shown e.g. in [10-16] and illustrated in the next Sections with the aid of some meaningful application example.

\section{INDENTATION TOOL}

$\mathrm{I}$ ndentation test represents a practical methodology for the characterization of traditional and innovative materials. With respect to more traditional experimental investigation, indentation can be performed on small material portion, does not require to extract laboratory specimens, can be performed even in situ, directly on the structural component, to continuous monitoring and diagnosis purposes. The outcome of instrumented indentation, namely the curves that represent the relationship between the penetration depth and the force exerted on the equipment tip, see Fig. 1 , reflect constitutive and fracture properties of the sampled material, though in an indirect way. Quantitative calibration of parameters entering the selected constitutive model can then be returned by inverse analysis procedures, which combine experimental data and the simulation of the laboratory test as shown, e.g., in $[9,17,18]$. The inverse analysis problem can be formulated as the minimisation, with respect to the unknown parameters, of a norm that quantifies the overall discrepancy between the measured quantities and the corresponding values computed through a mathematical or numerical model. Indentation test can be analysed in the large deformation regime by finite element (FE) approaches, as already done in previous analyses [10-16] or by alternative numerical techniques, like in [19,20]. Numerical simulation represents an useful tool also to the purpose of designing the experimental test, and selecting the most appropriate measurements, e.g. by sensitivity analysis [21].

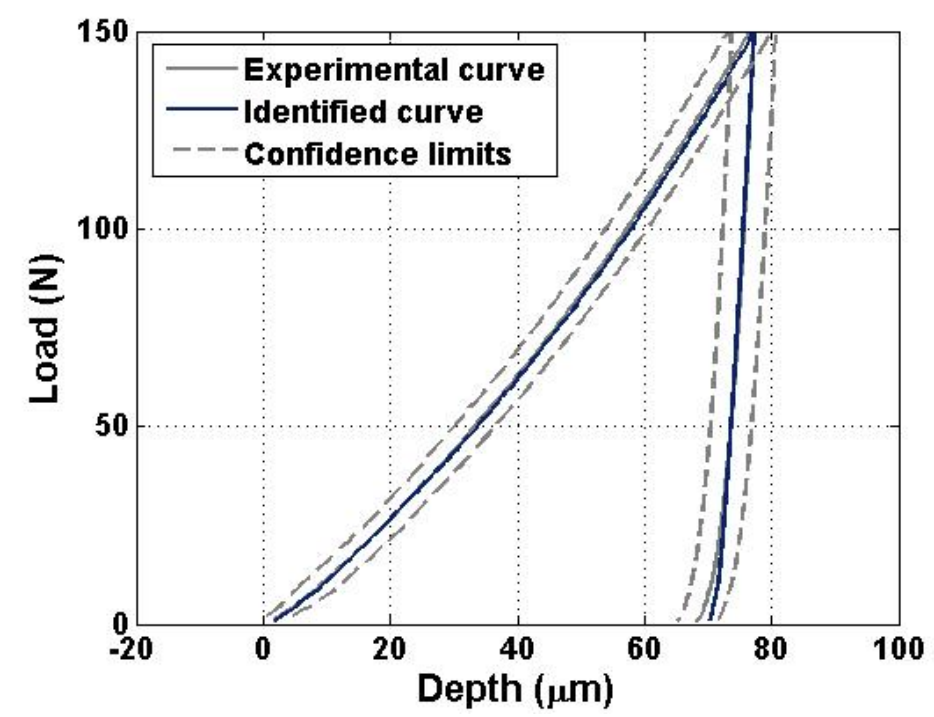

Figure 1: Comparison between the experimental mean indentation curves obtained from conical (Rockwell) tests on an $\mathrm{Al} / \mathrm{TiB}_{2}$ specimen with $50 \% \mathrm{TiB}_{2}$ weight content and the corresponding curves recalculated by a FE model of the test, supplemented by the identified material parameter set.

Let the indentation forces $F_{i}(i=1, \ldots, N)$ and the corresponding penetration depth $d_{M i}$ represent the experimental information collected from the mean indentation curves, visualised in Fig. 1. For any given value $F_{i}$, numerical analysis returns the corresponding penetration depth $d_{C i}(\mathbf{z})$ as a function of the constitutive parameters contained in the selected material or fracture model (elastic moduli, yield limits, fracture energy, ...), here collected by vector $\mathbf{z}$.

The discrepancy between quantities gathered from the experimental apparatus and from the simulation of the test can then be defined by the norm: 


$$
\omega(\mathbf{z})=\sum_{i=1}^{N}\left(\frac{d_{C i}(\mathbf{z})-d_{M i}}{w_{i}}\right)^{2}
$$

where $w_{i}$ represent weights on the displacement components $d_{M i}$, assumed to be proportional to the variances which quantifies the measurement discrepancies, also visualised in Fig. 1.

The optimum value of the sought parameters is represented by the entries of vector $\mathbf{z}_{\text {opt }}$, which corresponds to the minimum discrepancy. These values can be returned by a number of numerical methods implemented in widely available optimisation tools [22].

In this kind of application, the discrepancy function $\omega(\mathbf{z})$ is expected to be non-convex and to admit multiple minimum points, often almost equivalent from the engineering point of view, in the sense that the corresponding parameter values approximate to the same extent the available experimental data and return comparable representation of the real material behaviour, consistently with the selected constitutive model. The optimisation algorithm is hence run several times, starting from different initial parameter sets or, alternatively, evolutionary search techniques like genetic algorithms or soft computing methodologies are exploited; see, e.g., the review paper [20]. The computational efficiency of the simulation tool represents, hence, an important issue in this context.

\section{Bulk Material Properties}

$\Lambda$ ccording to a popular approach developed by Tamura et al. [23] for metal alloys and applied to different composite systems by a proper calibration of a characteristic 'stress transfer' parameter, the overall mechanical response of metal-ceramic composites is governed by the metal phase. Pressure-insensitive elastic-plastic laws, like the classical Hencky-Huber-Mises (HHM) model, are therefore mostly considered at the macro-scale [24-26] and supplemented by constitutive parameters (elastic modulus, yield limit, hardening coefficients), evaluated on the basis of some mixture theory that depends on volume fractions. In the proposal by Bocciarelli et al. [15], volume fractions govern the transition from HHM model toward Drucker-Prager (DP) constitutive law, capable to describe the mechanical behaviour of ceramics [14].

The elastic domain defined by the traditional DP yield criterion with linear isotropic hardening is represented by:

$$
f=\sqrt{\frac{1}{2} \sigma_{i j}^{\prime} \sigma_{i j}^{\prime}}+\alpha I_{1}-k-h \lambda \leq 0
$$

where: $\sigma_{i j}^{\prime}$ denote the components of the deviatoric stress tensor; $I_{1}$ represents the first stress invariant, i.e. the trace of the tensor collecting the stress components $\sigma_{i j} ; \lambda(>0)$ is the cumulative multiplier of the plastic deformations, which develop as $f=0$ and $\dot{f} \geq 0$ (the superimposed dot denotes a rate quantity); $\alpha, k$ and $b$ are constitutive parameters. Internal friction $a$ and initial cohesion $k$ depend on the initial tensile and compressive yield limits, $\sigma_{t 0}$ and $\sigma_{c 0}$, respectively, as follows:

$$
\alpha=\sqrt{3} \frac{\sigma_{c 0}-\sigma_{t 0}}{\left(\sigma_{c 0}+\sigma_{t 0}\right)}, \quad k=\frac{2}{\sqrt{3}} \frac{\sigma_{c 0} \sigma_{t 0}}{\left(\sigma_{c 0}+\sigma_{t 0}\right)}
$$

while parameter $b$ governs material hardening. HHM criterion can be recovered from DP model as the value of the internal friction coefficient $\alpha$ is set equal to zero.

The plastic rate components $\dot{\varepsilon}_{i j}^{p}$ of the strain tensor are assumed to develop orthogonally to a potential surface $g\left(\sigma_{i j}\right)$ as follows:

$$
\dot{\varepsilon}_{i j}^{p}=\dot{\lambda} \frac{\partial g}{\partial \sigma_{i j}}, \quad g\left(\sigma_{i j}\right)=\sqrt{\frac{1}{2} \sigma_{i j}^{\prime} \sigma_{i j}^{\prime}}+\beta I_{1}
$$

where $\beta$ represents the dilatancy coefficient. 
Parameters governing DP constitutive law have been inferred from available experimental information gathered from conical Rockwell indentation of $\mathrm{Al} / \mathrm{TiB}_{2}, \mathrm{Al} / \mathrm{ZrO}_{2}$ and $\mathrm{Cu} / \mathrm{Al}_{2} \mathrm{O}_{3}$ with about 40 to $50 \%$ weight metal content. Associative plasticity has been then introduced as reasonable hypothesis, so that $\alpha=\beta$ in relations (4).

Poisson's ratio has been a priori fixed due to the expected low influence of this parameter on indentation results; see [10, 14] and references therein. Then, the unknown overall material parameters to be returned by inverse analysis are: the elastic modulus $E$; the initial compression yield stress $\sigma_{c 0}$; the internal friction angle $a$ and the hardening modulus $b$.

Results listed in Tab. 1 concern the case of an $\mathrm{Al} / \mathrm{TiB}_{2}$ sample with $50 \% \mathrm{TiB}_{2}$ weight content produced by spark plasma sintering in a joint research project with Inasmet-Tecnalia (San Sebastian, Spain), partner of the KMM NoE [8]. The identified parameter values are given in terms of the average and of the standard deviation of several converged optimization solutions, which returned small similar values of $\omega\left(\mathbf{z}_{\text {opt }}\right)$ starting from different initial $\mathbf{z}$ vectors. The scatter on the value of the internal friction angle returned by the exploited inverse analysis procedure is relatively large, nevertheless $\alpha$ values were found much larger than zero in all considered situations, thus indicating that hydrostatic stress component plays a significant role in controlling the mechanical response of the considered metal-ceramic composites.

The accuracy of the selected material model and of the optimal parameter set obtained from the present identification procedure can be appreciated by the comparison between the experimental information and the corresponding recalculated curves, drawn in Fig. 1.

\begin{tabular}{ccccc}
\hline & $\begin{array}{c}\text { Elastic modulus } \\
\mathrm{E}[\mathrm{GPa}]\end{array}$ & $\begin{array}{c}\text { Initial yield limit in } \\
\text { compression } \\
\sigma_{c 0}[\mathrm{MPa}]\end{array}$ & $\begin{array}{c}\text { Internal friction } \\
\text { angle } \\
\operatorname{Arctan}(\alpha)\left[^{\circ}\right]\end{array}$ & $\begin{array}{c}\text { Hardening modulus } \\
\mathrm{h}[\mathrm{MPa}]\end{array}$ \\
$\mathrm{Al} / \mathrm{TiB}_{2}$ & $57.9( \pm 3.7)$ & $240.4( \pm 36.5)$ & $10.7( \pm 4.3)$ & $1032( \pm 91)$ \\
\hline
\end{tabular}

Table 1: Identified material properties after different initializations of the discrepancy minimisation algorithm for an $\mathrm{Al} / \mathrm{TiB}_{2}$ sample with $50 \% \mathrm{TiB}_{2}$ weight content.

The results of this research work showed that the overall material properties are strongly influenced by embedded defects and local damages, as earlier observed for different material systems [6]. Classical homogenization rules and mixture laws, which do not consider micro-structural details, fail in returning reliable quantitative prediction of constitutive parameters even in the elastic range.

\section{Fracture Properties}

$\mathrm{T}$ he brittleness of the composite materials under investigation is mitigated by the ductility of their metal phase. In fact, during fracture processes, large plastic deformations produce metal ligaments which bridge crack surfaces as shown e.g. in Fig. 2. This image is relevant to a fracture experiment performed at the Technical University Darmstadt (TUD), partner of the $\mathrm{KMM}$ Network, on a $\mathrm{Cu} / \mathrm{Al}_{2} \mathrm{O}_{3}$ composite with interpenetrating network structure [13].

Fracture can be induced in a brittle material sample to parameter identification purposes by the sharp corners of a Vickers pyramidal tip, as shown in Fig. 3 for the case of a pure zirconia $\left(\mathrm{ZrO}_{2}\right)$ specimen. Semi-empirical formulae based on linear elastic fracture mechanics (LEFM) permit to correlate the material toughness to the length of the cracks, measured from the centre of the imprint left by indentation $[27,28]$ which can be observed after unloading. However, experimental investigations show a systematic dependence of such estimated toughness values on the level of the applied indentation load. This result, inconsistent with LEFM assumptions, is likely originated by various error sources like the experimental difficulties in getting reliable measurement of the actual crack length and the influence of possible residual stresses, besides the existence of a crack-bridging zone at the crack tip [1, 4]. Furthermore, indentation results of brittle and quasibrittle materials are affected by big scatters and influenced by microstructure [29, 30]. These limitations can be mitigated by an identification approach based on inverse analysis, as introduced above, comparing results gathered from the experiment and from the test simulation.

Material separation during fracture propagation can be introduced in FE simulation of the indentation tests by interface elements, as shown e.g. in Fig. 4 and 5. The relationship between displacement discontinuities and the cohesive tractions transmitted across the fracture surface of metal-ceramic composites can be described by relatively simple and 
computational effective cohesive model originally proposed for mode I fracture for metals and bimetallic interfaces by Rose et al. [31], further extended in [32, 33] and widely employed in different application fields; see, e.g., [12, 14, 16, 37] and references therein.

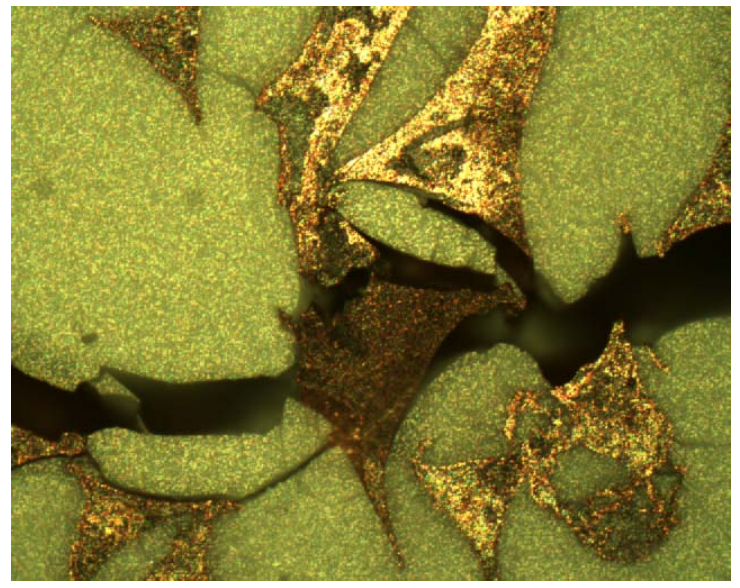

Figure 2: Detail of a fracture experiment on a $\mathrm{Cu} / \mathrm{Al}_{2} \mathrm{O}_{3}$ composite (courtesy of TUD)
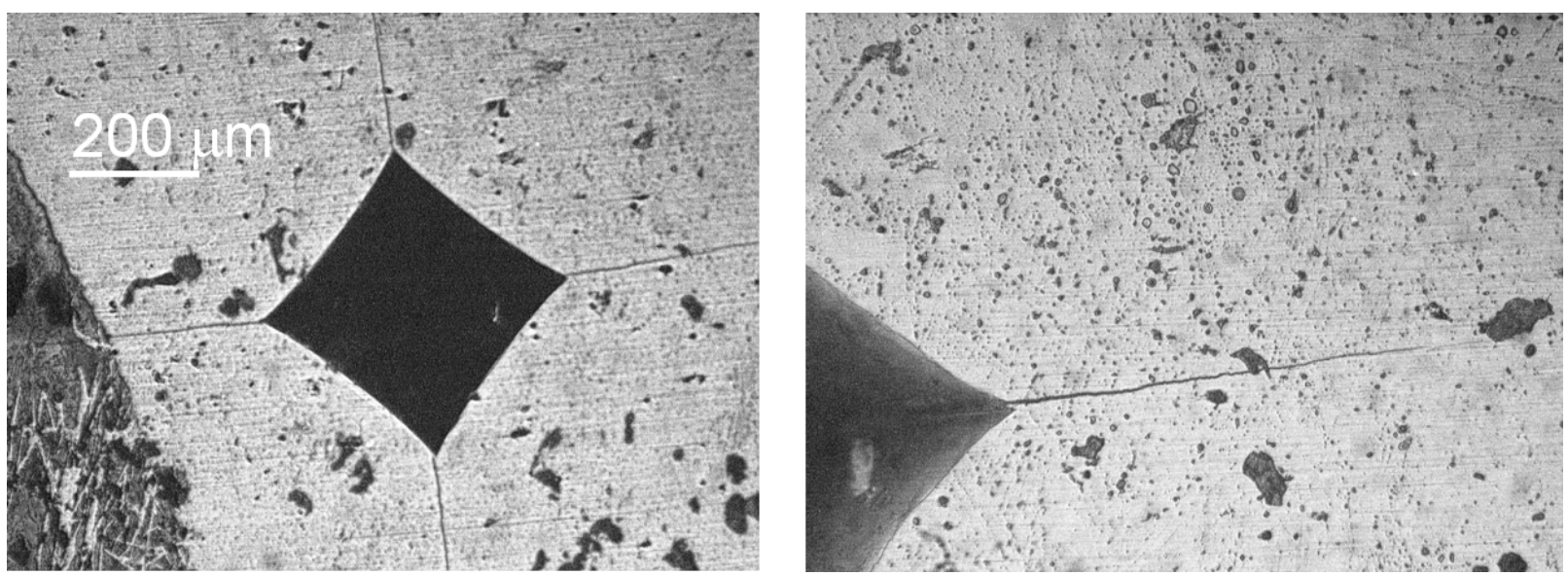

Figure 3: Result of Vickers indentation on a $\mathrm{ZrO}_{2}$ specimen.

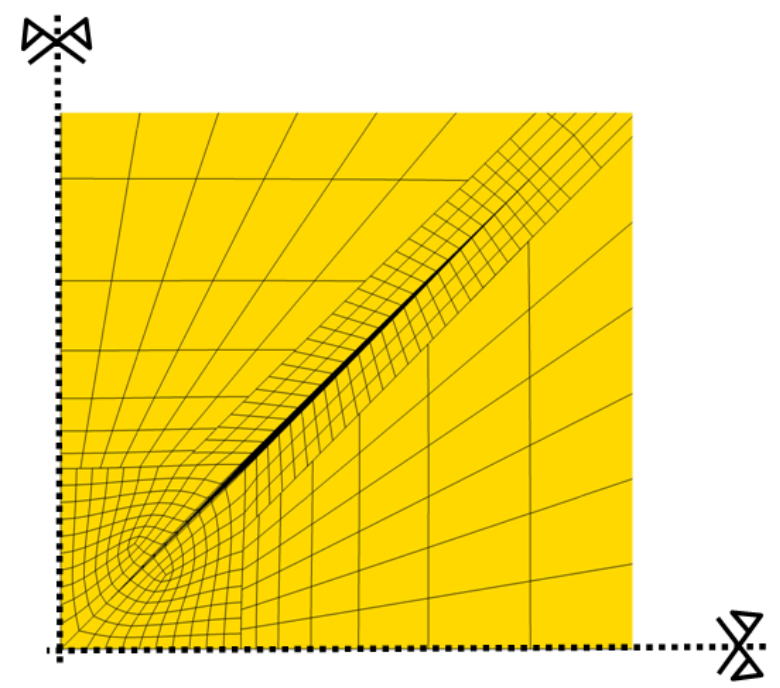

Figure 4: Simulation result of Vickers indentation on a $\mathrm{ZrO}_{2}$ specimen. 


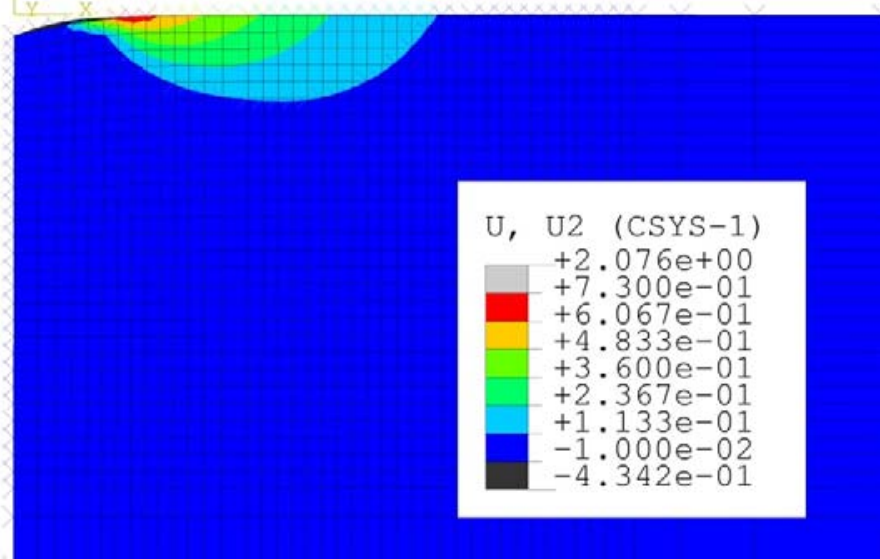

Figure 5: Contour map of the crack opening displacements of the half-penny shape fracture surface produced by indentation (simulation results)

According to this popular formulation, the normal cohesive stress $\sigma$ transferred during progressive mode I fracture processes can be described by the relationship:

$$
\sigma(w)=e \frac{\sigma_{c}}{w_{c}} w e^{-\frac{w}{w_{c}}}
$$

where: $e$ is the Neper number; $\sigma_{C}$ is the maximum cohesive normal traction; $w$ represents the opening displacement and $w_{C}$ is a characteristic value of it, associated to the fracture energy $G_{f}$ of the material by the formula:

$$
G_{f}=\int_{0}^{\infty} \sigma(w) d w=e \sigma_{c} w_{c}
$$

In mixed model fracture formulation, the opening displacement $w$ is replaced by a scalar measure of the displacement jump vector across the interface and a further parameter is introduced to describe the shear strength of the interface [32, 33].

Preliminary studies have shown that the indentation curve, the geometry of the residual imprint and the crack length reflect to different extent fracture properties like $\sigma_{C}$ and $G_{f}$, entering relation (5) and (6). The biggest sensitivity to a change in these parameters is shown by the fracture length but, in turn, the actual position of the crack tip is rather difficult to establish with the required precision. For this reason, information about the geometry of the residual imprint and in-plane displacement components on the specimen surface after fracture propagation can be acquired by several kinds of microscopes nowadays available on the market, possibly endowed with suitable image correlation tools, see e.g. $[35,36]$. These data can be exploited to enhance the identification of fracture properties through indentation test $[12,14$, 16], including them in the discrepancy function (1), compared to the corresponding computed quantities.

The application, now in progress, of reliable and robust identification procedures should also allow to verify the range of applicability of approaches based on modified mixture laws, proposed for fracture properties of metal-ceramic composites by Jin et al. [37], in analogy with [23].

\section{Closing REMARKS}

he envisaged increasing use of metal-ceramic composites for structural applications in the near future require adequate mechanical characterization, which can be better based on instrumented indentation and inverse analysis rather than on standard laboratory tests, due to difficulties and costs associated with material production and specimen extraction processes.

Recently developed parameter calibration methodologies, gathering experimental information from the measurement of the residual deformed configuration of the specimen after the test, besides from the indentation curves, are generally bound to enhance the identification of both bulk and fracture parameters. 
The application of these novel fast and reliable identification techniques can assist the selection of the constitutive models better suited to describe the behaviour of these composites and permits to appreciate the influence of micro-structural details on the overall mechanical characteristics. In fact, left porosity and micro defects generated by the production processes, generally neglected by material parameter prediction approaches based on classical homogenization rules, have strong influence on the properties of these quasi-brittle composites.

\section{ACKNOWLEDGEMENT}

$\mathrm{E}$ $\mathrm{U}$ financial support to the project 'Knowledge-based Multi-component Materials for durable and safe performance' (KMM-NoE, NMP3-CT-2004-502243) is gratefully acknowledged.

\section{REFERENCES}

[1] H. Prielipp, M. Knechtel, N. Claussen, S.K. Streiffer, H. Muellejans, M. Ruehle, J. Roedel., Mater. Sci. Eng., A197 (1995) 19.

[2] R.J. Moon, M. Tilbrook, M. Hoffman, A. Neubrand, J. Am. Ceramic Soc., 88 (2005) 666.

[3] S. Skirl, R. Krause, S.M. Wiederhorn, J. Roedel, Journal of the American Ceramic Society, 84 (2001) 2034.

[4] M. Basista, W. Węglewski, J. Theor. Appl. Mech., 44 (2006) 455.

[5] Z. Poniznik, V. Salit, M. Basista, D. Gross, Computat. Mater. Sci., 44 (2008) 813.

[6] H.A. Bruck, R.H. Rabin, J. Mater. Sci., 34 (1999) 2241.

[7] A.M. Klaska, T. Beck, A. Wanner, D. Lohe, Mater. Sci. Eng. A 501 (2009) 6.

[8] G. Bolzon, E.J. Chiarullo, P. Egizabal, C. Estournes, Mechanical modelling and characterization of aluminium-based metal matrix composites produced by spark plasma sintering, submitted.

[9] G. Stavroulakis, G. Bolzon, Z. Waszczyszyn, L. Ziemianski, in Comprehensive Structural Integrity, B. Karihaloo, R.O. Ritchie, I. Milne (eds), Elsevier Science Ldt, 3 (2003) 685-718.

[10] G. Bolzon, G. Maier, M. Panico, Int. J. Solids Struct., 41 (2004) 2957.

[11] M. Bocciarelli, G. Bolzon, G. Maier, Mech. Mat., 37 (2005) 855.

[12] G. Maier, M. Bocciarelli, G. Bolzon, R. Fedele, Int. J. Fract., 138 (2006) 47.

[13] M. Bocciarelli, G. Bolzon, Mater. Sci. Engng A, 448 (2007) 303.

[14] G. Bolzon, M. Bocciarelli, E.J. Chiarullo, in Applied Scanning Probe Methods XII Characterization, B. Bhushan, H. Fuchs (eds), Springer-Verlag, Heidelberg, (2008) 85.

[15] M. Bocciarelli, G. Bolzon, G. Maier, Computat. Mater. Sci., 43 (2008) 16.

[16] M. Bocciarelli, G. Bolzon, Int. J. Fract., 155 (2009) 1.

[17] H.D. Bui. Inverse Problems in the Mechanics of Materials: an Introduction, CRC Press, Boca Raton, FL (1994).

[18] Z. Mroz, G. Stavroulakis, Eds. Parameter Identification of Materials and Structures, Springer-Verlag, Berlin (2004).

[19] Z. Ostrowski, R.A. Bialecki, A.J. Kassab, Inv. Problems Sci. Eng., 16 (2008) 705.

[20] G. Maier, G. Bolzon, V. Buljak, T. Garbowski, B. Miller, in: Computer Methods in Mechanics - Lectures of the CMM 2009, Springer Verlag, Eidelberg (2009).

[21] M. Kleiber, H. Antúnez, T.D. Hien, P. Kowalczyk, Parameter sensitivity in non-linear mechanics. Theory and finite element computations, John Wiley \& Sons, Chichster (1997).

[22] The Math Works Inc. Matlab User's Guide and Optimization Toolbox, release 6.13, Natick, MA, USA (2004).

[23] I. Tamura, Y. Tomota, H. Ozawa, Proc. of the Third International Conference on Strength of Metals and Alloys, Volume 1, Institute of Metals, Cambridge (1973) 611.

[24] T. Nakamura, T. Wang, S. Sampath, Acta Mater., 48 (2000) 4293.

[25] A.E. Giannakopoulos, Int. J. Solids Structures, 39 (2002) 2495.

[26] Y. Gu, T. Nakamura, L. Prchlik, S. Sampath, J. Wallace, Mat. Sci. Eng. A, 345 (2003) 223.

[27] B.R. Lawn, A.G. Evans, D.B. Marshall, J. Am. Ceram. Soc., 63 (1980) 574.

[28] B.R. Lawn. Fracture of brittle solids. Cambridge University Press, Cambridge (1993).

[29] C. Ullner, A. Germak, H. Le Doussal, R. Morrell, T. Reich, W. Vandermeulen, J. Eur. Ceramic Soc. 21 (2001) 439.

[30] L.L. Mishnaevsky Jr., Acta Materialia, 52 (2004) 4177.

[31] J.H. Rose, J. Ferrante, J.R.Smith, Phys. Rev. Letters, 47 (1981) 675. 
[32] X.P. Xu, A. Needleman, Modelling Sim. Mat. Sci. Eng., 1 (1993) 111.

[33] M. Ortiz, S. Suresh, ASME J. of Appl. Mech., 60 (1993) 77.

[34] P. Liu, Y.W. Zhang, K.Y. Zeng, C. Lu, K.Y. Lam, Eng. Fract. Mech., 74 (2007) 1118.

[35] D. Vogel, A. Gollhardt, B. Michel, Sensors and Actuators A, 99 (2002) 165.

[36] F. Hild, S. Roux, Strain, 42 (2006) 69.

[37] Z. H. Jin, G.H. Paulino, R.H. Dodds Jr., Eng. Fract. Mech., 70 (2003) 1885. 